TRANSACTIONS OF THE

AMERICAN MATHEMATICAL SOCIETY

Volume 351, Number 4, April 1999, Pages 1551-1573

S 0002-9947(99)02077-2

\title{
ENTROPY AND PERIODIC POINTS FOR TRANSITIVE MAPS
}

\author{
LL. ALSEDÀ, S. KOLYADA, J. LLIBRE, AND L` ${ }^{\vee}$ SNOHA \\ Dedicated to the memory of Wiestaw Szlenk
}

\begin{abstract}
The aim of this paper is to investigate the connection between transitivity, density of the set of periodic points and topological entropy for low dimensional continuous maps. The paper deals with this problem in the case of the $n$-star and the circle among the one-dimensional spaces and in some higher dimensional spaces. Particular attention is paid to triangular maps and to extensions of transitive maps to higher dimensions without increasing topological entropy.
\end{abstract}

\section{INTRODUCTION AND MAIN RESULTS}

Recently, several authors became interested in studying the dynamics of maps of the interval, the circle, the $Y$ and the $n$-star (see for instance [6], [15], [5], [11]) and the dynamics of tree maps (see for instance [12], [34], [8]). The interest in studying maps on such one-dimensional spaces is due to the fact that for maps on manifolds with an invariant foliation of codimension one, the corresponding quotient map turns out to be defined in general on a graph. Furthermore, the dynamics of pseudo-Anosov homeomorphisms on a surface can be essentially reduced to the analysis of some special graph maps (see e.g. [27]). Finally, a graph map sometimes imitates the behavior of a smooth map (flow) in a neighborhood of a hyperbolic attractor (see for instance [43]). One of the main reasons to study the transitive one-dimensional maps is the fact that the invariant subsets of transitive maps are models for the $\omega$-limit sets of arbitrary maps on which the entropy is positive (see $[20])$.

Triangular maps are close to one-dimensional maps in the sense that some important dynamical features extend to triangular maps. On the other hand, they already display other important properties which are typical for higher dimensional maps and cannot be found in the one-dimensional maps (see for instance [29], [4], $[30],[2])$. We will show that this dualism can also be found in the study of the transitive triangular maps.

Let $f: X \longrightarrow X$ be a continuous map of a compact metric space. We say that $f$ is (topologically) transitive if for any two non-empty open sets $U$ and $V$ in $X$, there is a nonnegative integer $k$ such that $f^{k}(U) \cap V \neq \emptyset$. If $X$ has no isolated points, then the above definition is equivalent to the following one. The map $f$ is transitive if it has a dense orbit, i.e. if there exists $x \in X$ such that the orbit of $x$,

Received by the editors September 20, 1995 and, in revised form, November 26, 1996.

1991 Mathematics Subject Classification. Primary 58F11, 34C35, 54H20.

Key words and phrases. Transitive maps, topological entropy, periodic points, triangular maps.

(c)1999 American Mathematical Society 
$\left\{f^{n}(x): n=0,1,2, \ldots\right\}$, is dense in $X$. If every orbit of $f$ is dense, the map $f$ is called minimal. A set $M \subset X$ is called minimal if it is non-empty, closed, invariant (i.e., $f(M) \subset M$ ) and no proper subset of $M$ satisfies these three properties.

Let $f: X \longrightarrow X$ be a map. A point $x \in X$ is periodic if $f^{n}(x)=x$ for some $n \in \mathbb{N}$. The smallest $n$ satisfying this equality is called the period of $x$.

Let $I$ be a closed interval of the real line. It is well-known that the set of all periodic points of a transitive continuous map $f: I \longrightarrow I$ is dense in $I$ (see [39], [14] and [15]). The following theorem extends this result to transitive maps on a more general class of topological spaces.

Let $X$ be a connected space and let $J$ be an open subset of $X$. We say that $J$ is a disconnecting interval of $X$ if it is homeomorphic to an open interval of the real line and, for each $x \in J, X \backslash\{x\}$ has exactly two connected components. Note that any open connected subset of $J$ is also a disconnecting interval.

Theorem 1.1. Let $X$ be a connected space with a disconnecting interval and let $f: X \longrightarrow X$ be a transitive map. Then the set of all periodic points of $f$ is dense in $X$.

The fact that the set of periodic points of a transitive map is dense was proved when the space is a graph by Blokh in [20], by using a spectral decomposition.

The topological entropy of a continuous map $f$ from a compact space into itself, denoted by $h(f)$, was introduced in [1] (see also [24]). An equivalent definition using $(n, \varepsilon)$-separated sets can be found in [21].

A tree is any space which is uniquely arcwise connected and homeomorphic to the union of finitely many copies of the unit interval. If $T$ is a tree and $x$ is a point of $T$, the valence of $x$ is the number of components of $T \backslash\{x\}$. A point of valence 1 is called an endpoint and a point of valence greater than 2 is called a branching point. A continuous map $f: T \longrightarrow T$ is called a tree map.

From Blokh we know that if $f: T \longrightarrow T$ is a transitive tree map, then $f$ has positive topological entropy; and that if an interval map $f: I \longrightarrow I$ is transitive, then $h(f) \geq(\log 2) / 2$ (see [20] and [18], respectively). An interesting question is to obtain lower bounds for the topological entropy of transitive tree maps which are not interval maps. Franks and Misiurewicz in [27] studied a special class of transitive tree maps. For such maps they proved that $(\log 2) / n$ is a lower bound of the topological entropy, where $n$ is the number of endpoints of the tree. Fehrenbach and Los in [25] consider a similar problem for the transitive tree maps obtained from pseudo-Anosov diffeomorphisms of the disk. The bound of the topological entropy they get in that case is $(\log (1+\sqrt{2})) / n$.

We extend the results of Blokh, Franks and Misiurewicz and Fehrenbach and Los to the transitive $n$-star maps. The $n$-star $X_{n}$ is the subspace of the complex plane which is most easily described as the set of all complex numbers $z$ such that $z^{n}$ is in the unit interval $[0,1]$, i.e. a central point (the origin) with $n$ copies of the interval $[0,1]$ attached to it. Notice that the 1-star and the 2-star are homeomorphic. So, in what follows we only consider the $n$-star with $n \geq 2$. For $n \geq 2$ we denote the origin of the $n$-star by $b$. Note that when $n>2, b$ is the unique branching point of the $n$-star. Any continuous map from an $n$-star into itself will be called an $n$-star map.

Theorem 1.2. Let $f$ be a transitive $n$-star map. Then the following statements hold. 
(a) If $f(b) \neq b$, then $h(f) \geq(\log 2) / 2$.

(b) If $f(b)=b$, then $h(f) \geq(\log 2) / n$. Moreover, there exists a transitive $n$-star map $g$ such that $g(b)=b$ and $h(g)=(\log 2) / n$.

From Theorem 3.2 and Example 3.8 one can see that the lower bound for the entropy given in Theorem 1.2(a) is the best possible when $n \in\{2,3\}$. It is not known by the authors whether this lower bound is the best possible for $n>3$.

From Theorem 1.2 it follows that each transitive $n$-star map $f$ satisfies that $h(f) \geq(\log 2) / n$. This fact was extended recently in [7] obtaining lower bounds of the topological entropy for any transitive tree map.

Let $\mathbb{S}^{1}$ be the circle. A continuous map $f: \mathbb{S}^{1} \longrightarrow \mathbb{S}^{1}$ is called a circle map. Now we want to obtain lower bounds of the topological entropy for transitive circle maps. It is known that the irrational rotations of the circle are transitive maps without periodic points and with zero topological entropy. In fact Auslander and Katznelson in [10] proved that any transitive circle map without periodic points is topologically conjugate to an irrational rotation and, hence, it has zero topological entropy.

Coven and Mulvey in [23] extended Theorem 1.1 to the circle proving that for transitive circle maps with periodic points the set of all periodic points is dense in $\mathbb{S}^{1}$. Moreover, Blokh in [20] showed that transitive circle maps with periodic points have positive topological entropy. We obtain the best lower bounds of the topological entropy for the transitive circle maps with periodic points depending on the degree of the map under consideration.

Theorem 1.3. Let $f: \mathbb{S}^{1} \longrightarrow \mathbb{S}^{1}$ be a transitive circle map of degree $d$. Then the following statements hold.

(a) If $|d|>1$, then $h(f) \geq \log |d|$.

(b) If $d=0$, then $h(f) \geq \log 2$.

(c) If $d=-1$, then $h(f) \geq(\log 3) / 2$.

(d) If $d=1$ and $f$ has periodic points, then $h(f)>0$.

(e) If $d=1$ and $f$ has no periodic points, then $h(f)=0$.

Moreover, there exist transitive circle maps $f_{0}, f_{-1}$ and $f_{d}$ with degree $0,-1$ and $d \in \mathbb{Z} \backslash\{-1,0,1\}$ respectively, such that $h\left(f_{0}\right)=\log 2, h\left(f_{-1}\right)=(\log 3) / 2$ and $h\left(f_{d}\right)=\log |d|$.

Theorem 1.3(d,e) were proved in [20] and [10] respectively (see also [34]), and Theorem 1.3(a) was proved in [17] (see also [6]).

A natural question is: Does there exist a positive lower bound for the topological entropy of a transitive circle map of degree one with periodic points? The negative answer is given by the following folk knowledge result. However, we provide a proof of it because we have not been able to find an explicit reference for this result.

Proposition 1.4. The infimum of the (positive) topological entropies for the transitive circle maps of degree 1 with periodic points is zero.

Now it is natural to ask what is the connection between transitivity, topological entropy and the density of the set of periodic points in higher dimensions. One of the possible approaches is to try to use known results on one-dimensional transitive maps to get corresponding results for higher dimensional maps. Of course, the problem is nontrivial and one can hardly expect that the one-dimensional results 
could be simply carried over to higher dimensions. We use triangular maps to get some insight on these problems.

Let us recall that the triangular maps are defined as follows. Let $\left(X_{i}, \rho_{i}\right)$ be metric spaces for $i=1,2, \ldots, n$. We assume that the set $\prod_{i=1}^{n} X_{i}$ is endowed with the metric $\rho(x, y)=\max _{1 \leq i \leq n} \rho_{i}\left(x_{i}, y_{i}\right)$, where $x=\left(x_{1}, \ldots, x_{n}\right)$ and $y=$ $\left(y_{1}, \ldots, y_{n}\right)$. A map $F$ from $\prod_{i=1}^{n} X_{i}$ into itself is called triangular if it is continuous and is of the form

$$
F\left(x_{1}, x_{2}, \ldots, x_{n}\right)=\left(F_{1}\left(x_{1}\right), F_{2}\left(x_{1}, x_{2}\right), \ldots, F_{n}\left(x_{1}, x_{2}, \ldots, x_{n}\right)\right),
$$

where $\left(x_{1}, x_{2}, \ldots, x_{n}\right) \in \prod_{i=1}^{n} X_{i}$. So the triangularity means continuity and the dependence of the $i$-th component map only on the first $i$ variables for every $i$. The set of all triangular maps from $\prod_{i=1}^{n} X_{i}$ into itself will be denoted by $\mathcal{C}_{\triangle}\left(\prod_{i=1}^{n} X_{i}\right)$. When all the spaces $X_{i}$ are the interval $I$, instead of $\mathcal{C}_{\triangle}\left(\prod_{i=1}^{n} X_{i}\right)$, we will simply write $\mathcal{C}_{\triangle}\left(I^{n}\right)$ and we will always understand that a map $F \in \mathcal{C}_{\triangle}\left(I^{n}\right)$ is triangular at all levels. That is, it satisfies $(*)$. If $n \geq 2$, we can always think on the map $F$ as a triangular map defined on the "rectangle" $\left(\prod_{i=1}^{n-1} X_{i}\right) \times X_{n}$ by $F(x, y)=$ $(f(x), g(x, y))$, where $x=\left(x_{1}, x_{2}, \ldots, x_{n-1}\right), y=x_{n}$ and

$$
\begin{aligned}
f(x) & =\left(F_{1}\left(x_{1}\right), F_{2}\left(x_{1}, x_{2}\right), \ldots, F_{n-1}\left(x_{1}, x_{2}, \ldots, x_{n-1}\right)\right), \\
g(x, y) & \left.=F_{n}\left(x_{1}, x_{2}, \ldots, x_{n}\right)\right) .
\end{aligned}
$$

Here $f$ is also a triangular (and not an arbitrary continuous) map from $\prod_{i=1}^{n-1} X_{i}$ into itself and $g(x, y)$ is a map from $\left(\prod_{i=1}^{n-1} X_{i}\right) \times X_{n}$ to $X_{n}$.

Let $I$ be a compact interval of the real line (say the unit interval $[0,1]$ ) with the usual metric and let $(X, \rho)$ be a compact metric space. We will denote by $\mathcal{C}(X)$ the set of all continuous maps from $X$ into itself. Also, each map $F \in \mathcal{C}_{\triangle}(X \times I)$ will be written as $F(x, y)=(f(x), g(x, y))$ where $f \in \mathcal{C}(X)$ and $g$ is a continuous map from $X \times I$ to $I$. Instead of $g(x, y)$ we can also write $g_{x}(y)$, where $g_{x}: I \longrightarrow I$ is a family of continuous maps depending continuously on $x \in X$. The map $f$ is called the basis map of $F$ and the maps $g_{x}$ are called the fiber maps. The map $F$ splits the rectangle $X \times I$ into one-dimensional fibers $I_{x}=I(x)=\{x\} \times I$ for $x \in X$ such that each fiber is mapped by $F$ into a fiber. Though, in this sense, triangular maps are similar to the basis ones (more precisely, $F$ is an extension of $f$ ), there are essential differences in the dynamics between them for the case $X=I$ (see [33], [30], [31], [2] and [26]).

To study the topological entropy of triangular maps the Bowen's definition [21] is more useful than the Adler, Konheim and McAndrew's one. Let $(X, \rho)$ be a compact metric space and let $f$ be a continuous map from $X$ into itself. A subset $E$ of $X$ is called $(n, \varepsilon)$-separated if for every two different points $x, y \in E$ there exists $0 \leq j<n$ with $\rho\left(f^{j}(x), f^{j}(y)\right)>\varepsilon$. A set $E_{1} \subset X(n, \varepsilon)-$ spans another set $K \subset X$ provided that for each $x \in K$ there is $y \in E_{1}$ for which $\rho\left(f^{j}(x), f^{j}(y)\right) \leq$ $\varepsilon$ for all $0 \leq j<n$. For a compact set $K \subset X$ let $s_{n}(\varepsilon, K)$ be the maximal possible cardinality of an $(n, \varepsilon)$-separated set $E$ contained in $K$ and let $r_{n}(\varepsilon, K)$ be the minimal possible cardinality of a set $E_{1}$ which $(n, \varepsilon)$-spans $K$ (we will write $s_{n}(\varepsilon, K, f)$ and $r_{n}(\varepsilon, K, f)$ if we wish to stress the dependence on $\left.f\right)$. Further, let

$$
s(\varepsilon, K, f)=\limsup _{n \rightarrow \infty} \frac{1}{n} \log s_{n}(\varepsilon, K, f)
$$


and

$$
r(\varepsilon, K, f)=\limsup _{n \rightarrow \infty} \frac{1}{n} \log r_{n}(\varepsilon, K, f) .
$$

Then the entropy of $f$ on the set $K$ is defined by

$$
h_{\rho}(f, K)=\lim _{\varepsilon \rightarrow 0} s(\varepsilon, K, f)=\lim _{\varepsilon \rightarrow 0} r(\varepsilon, K, f)
$$

and the topological entropy of $f$ by

$$
h(f)=h_{\rho}(f, X) .
$$

Topological entropy of triangular maps of the square $I^{2}$ has been studied in [30] and [2].

Let $F=\left(f, g_{x}\right) \in \mathcal{C}_{\triangle}(X \times I)$ and suppose that $X \times I$ is endowed with the product metric, $d$, of $\rho$ in $X$ with the Euclidean metric in $I$. We define $h_{f}(F)=$ $\sup _{x \in X} h_{d}\left(F, I_{x}\right)$, where $h_{d}\left(F, I_{x}\right)$ is called the entropy of $F$ on the fiber $I_{x}$. Then, from Bowen's formula (see [21]), one gets

$$
\max \left\{h(f), h_{f}(F)\right\} \leq h(F) \leq h(f)+h_{f}(F),
$$

where $h(f)$ and $h(F)$ denote the topological entropy of $f$ and $F$, respectively. So, if all the fiber maps $g_{x}$ are monotone, then $h_{d}\left(F, I_{x}\right)=0$ for any $x$ (see [32]). Thus $h(F)=h(f)$.

If $F=\left(f, g_{x}\right) \in \mathcal{C}_{\triangle}(X \times I)$ is transitive, then evidently its basis map $f$ is transitive. In the particular case $X=I$ this means that $h(F) \geq h(f) \geq(1 / 2) \log 2$. For maximal cardinalities of $(n, \varepsilon)$-separated sets we trivially have $s_{n}(\varepsilon, X \times I, F) \geq$ $s_{n}(\varepsilon, X, f)$. One could expect that the rectangle $X \times I$ is (at least in the case $X=I$ ) sufficiently "large" when compared with the set $X$ to ensure that $s_{n}(\varepsilon, X \times I, F)$ is sufficiently larger than $s_{n}(\varepsilon, X, f)$ to give $h(F)>h(f)$. We show that usually (particularly if $X=I$ ) this is not true. In fact, we prove by the category method (see, e.g., [37]) the following result.

Theorem 1.5. Let $(X, \rho)$ be a compact metric space and let $f \in \mathcal{C}(X)$ be a transitive map which is not minimal. Then the map $f$ can be extended to a map $F \in \mathcal{C}_{\triangle}(X \times I)$ (i.e., $f$ is the basis map of $F$ ) in such a way that $F$ is transitive and has the same entropy as $f$.

Remark 1.6. Some authors define topological transitivity as the existence of a dense orbit. However, if one replaces in Theorem 1.5 the notion of transitivity by the existence of a dense orbit, then the theorem obtained in such a way would no longer be true as the following example shows. Take $X=\{a, b\}$, with $a \neq b$ and $f(a)=f(b)=b$. Then the point $a$ has a dense orbit and $f$ is not minimal but there is no map $F \in \mathcal{C}_{\triangle}(X \times I)$ which is an extension of $f$ and has a dense orbit. Of course, after adding the assumption that $X$ does not have isolated points the new theorem will become equivalent to Theorem 1.5 (see Section 1).

The assumption in Theorem 1.5 that $f$ is not minimal is a technical one but is essentially used in the proof. It is possible that the theorem still holds true without this assumption.

Theorem 1.5, being proved by the category method, does not give explicit examples of transitive triangular maps whose entropy is not greater than the entropy of their basis maps. In the particular case when $X=I$, we give such an explicit example. Moreover, the example shows that a transitive triangular map of the square $I^{2}$ need not have a dense set of periodic points. 
Theorem 1.7. The map

$$
F:(x, y) \longrightarrow\left(1-|2 x-1|, y^{\exp (x-\beta)}\right),
$$

where $\beta$ is any irrational number from $(0,2 / 3)$, is a transitive map from $\mathcal{C}_{\triangle}\left(I^{2}\right)$ with topological entropy $h(F)=\log 2$ (the same as the entropy of the basis map) such that the set of periodic points is contained in $I \times\{0,1\}$ (and hence is nowhere dense in $I^{2}$ ).

Remark 1.8 (Due to A. N. Sharkovsky). The map $F$ from Theorem 1.7 has another interesting property. First we note that since the basis map is the standard tent map, by the Ergodic Theorem, for almost all (in the sense of Lebesgue measure) points $x \in I$ we have $\lim _{n \rightarrow \infty} \frac{1}{n} \sum_{i=0}^{n-1} f^{i}(x)=1 / 2$. Further, an easy calculation shows that $F^{n}(x, y)=\left(f^{n}(x), y^{\exp \left(\sum_{i=0}^{n-1} f^{i}(x)-n \beta\right)}\right)$ for $n=1,2, \ldots$ So we get the following result for the $\omega$-limit sets $\omega_{F}(x, y)$ of points $(x, y) \in I^{2}$. If $\beta<1 / 2$ (respectively $\beta>1 / 2$ ), then for almost all (in the sense of Lebesgue measure) points $(x, y) \in I^{2}, \omega_{F}(x, y) \subset I \times\{0\}$ (respectively $\left.\omega_{F}(x, y) \subset I \times\{1\}\right)$. On the other hand it is well known that almost all (in the sense of Lebesgue measure) points $x \in I$ have dense orbits in $I$, i.e., $\omega_{f}(x)=I$. Therefore, if $\beta<1 / 2$ (respectively $\beta>1 / 2$ ), then for almost all points $(x, y) \in I^{2}, \omega_{F}(x, y)=I \times\{0\}$ (respectively $\left.\omega_{F}(x, y)=I \times\{1\}\right)$. More precisely, this is true for all points $(x, y)$ where $x$ belongs to a full Lebesgue measure subset of $I$ and, respectively, $y \in[0,1)$ or $y \in(0,1]$.

As a consequence of the above mentioned fact we immediately get that the set $I \times\{0\}$ if $\beta<1 / 2$ or the set $I \times\{1\}$ if $\beta>1 / 2$ is the Milnor attractor in the sense of Lebesgue measure for the dynamical system $\left(F, I^{2}\right)$ where $F$ is the map from Theorem 1.7 (see [35] for the definition of the Milnor attractor).

Theorem 1.5 will be used to prove the first three statements of the following result.

Theorem 1.9. The following statements hold.

(a) Let $(X, \rho)$ be a compact metric space such that there are no minimal maps in $\mathcal{C}(X)$. Then

$$
\begin{aligned}
& \inf \{h(f): f \in \mathcal{C}(X) \text { is transitive }\} \\
& \quad=\inf \left\{h(F): F \in \mathcal{C}_{\triangle}(X \times I) \text { is transitive }\right\}
\end{aligned}
$$

and if one of these infima is minimum, then so is the other.

(b) For every $n=2,3, \ldots$,

$$
\begin{aligned}
& \min \left\{h(F): F \in \mathcal{C}_{\triangle}\left(I^{n}\right) \text { is transitive }\right\} \\
& \quad=\min \{h(f): f \in \mathcal{C}(I) \text { is transitive }\}=(1 / 2) \log 2 .
\end{aligned}
$$

(c) For every $n=2,3, \ldots$, there are transitive maps from $\mathcal{C}\left(I^{n}\right)$ with arbitrarily small positive topological entropies. Consequently,

$$
\inf \left\{h(f): f \in \mathcal{C}\left(I^{n}\right) \text { is transitive and } h(f)>0\right\}=0 .
$$

(d) There exists a transitive orientation preserving homeomorphism of $I^{2}$ with zero topological entropy. Consequently,

$$
\min \left\{h(f): f \in \mathcal{C}\left(I^{2}\right) \text { is transitive }\right\}=0 .
$$

(e) For every $n=2,3, \ldots$,

$$
\min \left\{h(f): f \in \mathcal{C}\left(I^{n}\right) \text { is transitive }\right\}=0 .
$$


We do not exclude that statements (c-e) of the above theorem are known but we are unable to give a precise reference ${ }^{1}$.

The paper is organized as follows. Theorems 1.1 and 1.2 are proved in Sections 2 and 3, respectively. Theorems 1.3 and 1.4 in Section 4. Lastly, Theorems 1.5, 1.7 and 1.9 are proved in Sections 5, 6 and 7, respectively.

Acknowledgements. Ll. Alsedà and J. Llibre have been partially supported by the DGICYT grant number PB93-0860, S. Kolyada by the ISF grant number U6G000 and L. Snoha by the Slovak grant agency grant numbers 1/338/92-94 and 1/1470/94-96. S. Kolyada and L. Snoha thank Centre de Recerca Matemàtica and S. Kolyada also Matej Bel University for the invitation and kind hospitality. The support of these institutions enabled us to do most of the work on this paper and is gratefully acknowledged. The authors thank A. Blokh, R. Hric, M. Chlebík, M. Lyubich, M. Misiurewicz, A.N. Sharkovsky, J. Smítal and J. Wośko for their useful comments. They especially thank B. Sivák who solved a problem concerning sequences of zeros and ones [42] which enabled them to finish an old version of the proof of Theorem 1.7. Moreover, the authors are greatly indebted to the referee for extremely careful reading of the paper and many clever suggestions which improved the writing and some results of the paper and drastically shortened the proof of Theorem 1.7.

\section{Periodic points and transitive tree maps}

The goal of this section is to prove Theorem 1.1. To this end we will need the following definition and lemma.

Let $X$ be a connected space and let $J$ be a disconnecting interval of $X$. We assume that $J$ is endowed with a linear ordering $\leq$. Given $x \in J$ and $y \in X$ such that $x \neq y$ we write $x<y$ (respectively $x>y$ ) if and only if there exists $z \in J$ such that $x<z$ (respectively $x>z$ ) and $z$ and $y$ belong to the same connected component of $X \backslash\{x\}$.

Lemma 2.1. Let $X$ be a connected space with a disconnecting interval $J$ and let $f: X \longrightarrow X$ be a continuous map. Assume that there exist $x, y \in J$ and $n, m \geq 1$ such that $f^{n}(x), f^{m}(y) \in J, f^{n}(x)<x$ and $f^{m}(y)>y$. Then $f$ has a periodic point in the convex hull of $\left\{x, y, f^{n}(x), f^{m}(y)\right\}$.

Proof. Assume first that there exists $k>1$ such that $f^{k n}(x)>f^{n}(x)$. Without loss of generality we can assume that $k$ is the smallest possible satisfying this property. Set $j=(k-1) n$. Then $f^{j}(x) \leq f^{n}(x)<x$ and $f^{j}\left(f^{n}(x)\right)=f^{k n}(x)>x$. Therefore, since the interior of the convex hull of $\left\{x, f^{n}(x)\right\}$ is a disconnecting interval, $f^{j}$ has a fixed point between $x$ and $f^{n}(x)$. Consequently $f$ has a periodic point in $J$. In a similar way, if there exists $l>1$ such that $f^{l m}(y)<f^{m}(y)$, we are done.

Now we can assume that $f^{k n}(x) \leq f^{n}(x)$ and $f^{k m}(y) \geq f^{m}(y)$ for all $k>1$. In particular we have $f^{m n}(x) \leq f^{n}(x)<x$ and $f^{m n}(y) \geq f^{m}(y)>y$. Therefore, $f^{m n}$ has a fixed point between $x$ and $y$. This completes the proof.

Proof of Theorem 1.1. Let $K$ be a disconnecting interval in $X$ and let $U$ be a nonempty open subset of $X$. Since $f$ is transitive, there exists $k$ such that $f^{k}(K) \cap U \neq$

\footnotetext{
${ }^{1}$ According to the referee of the paper, these three assertions are essentially known and they could be deduced from [9] and [28] (even for $C^{\infty}$ diffeomorphisms). Nevertheless, since in these two papers the notion of topological entropy is not mentioned at all and our proofs are rather short, we decided to include them into the paper.
} 
$\emptyset$. Therefore, $f^{-k}(U) \cap K$ is a non-empty open subset of $K$. Let $J$ be an open subinterval of $f^{-k}(U) \cap K$ and let $a, b, c, d \in J$ be such that $a<b<c<d$. By the transitivity of $f$, there exist $x, y \in(b, c)$ and $n, m \geq 1$ such that $a<f^{n}(x)<b$ and $c<f^{m}(y)<d$. Thus, in view of Lemma 2.1, there exists a periodic point $z$ of $f$ in $J$. Hence, the periodic point $f^{k}(z)$ belongs to $U$. Consequently the set of periodic points of $f$ is dense in $X$.

\section{ENTROPY AND TRANSITIVE $n-$ STAR MAPS}

The aim of this section is to prove Theorem 1.2. We start with some preliminary results.

The following proposition, in the particular case of transitive interval maps, follows from [13] (see also Proposition 42 of Chapter VI of [15]). In fact, this result follows from a more general theorem of Blokh (for non-connected graphs) stated in [20] and proved in [19]. Recently, this proposition and Blokh's result have been extended to locally connected compact metric spaces in [3].

A closed subtree of a tree is called non-degenerate if it is different from a point.

Proposition 3.1. Let $f: T \longrightarrow T$ be a transitive tree map. Then exactly one of the following two statements holds:

(a) $f^{s}$ is transitive for all $s \geq 1$.

(b) There exist $k>1$, a fixed point $y$ of $f$ of valence larger than or equal to $k$ and non-degenerate closed subtrees $T_{1}, \ldots, T_{k}$ of $T$ such that $T=\bigcup_{i=1}^{k} T_{i}$, $T_{i} \cap T_{j}=\{y\}$ for all $i \neq j, f\left(T_{i}\right)=T_{i+1}$ for $i=1, \ldots, k-1$ and $f\left(T_{k}\right)=T_{1}$.

Proposition 3.1 enables us to split the proof of Theorem 1.2 into two subcases according to the fact that $f^{s}$ is transitive for all $s \geq 1$ or not.

A subset $J$ of a tree $T$ is called an interval if there exists a homeomorphism $h: I \longrightarrow J$, where $I$ is $[0,1],(0,1],[0,1)$ or $(0,1)$. The set $h((0,1))$ is called the interior of $J$. If $I=[0,1]$, the interval $J$ is called closed; if $I=(0,1)$, it is called open. We note that it may happen that an open interval is not an open set in $T$ and that the interior of this interval does not coincide with its interior in the topology of $T$.

A tree map $f: T \longrightarrow T$ is called turbulent if there are closed subintervals $J$ and $K$ of $T$ with disjoint interiors such that $f(J) \cap f(K) \supset J \cup K$. From Remark 4.3.4 of [6] we get that if a tree map $f$ is turbulent, then $h(f) \geq \log 2$.

The next theorem, which will be used later, is well-known (see Lemma 3.3, Theorem 3.4 and Corollary 3.6 of [16]).

Theorem 3.2. Let $f: I \longrightarrow I$ be a transitive interval map.

(a) If $f$ is not turbulent, then $f$ has a unique fixed point which is not an endpoint.

(b) The topological entropy of $f$ satisfies $h(f) \geq(\log 2) / 2$.

Moreover, there exists a transitive interval map g such that $h(g)=(\log 2) / 2$ having a unique fixed point which is not an endpoint.

Notice that Theorem 3.2 proves Theorem 1.2 in the case of interval maps. Next we shall concentrate on $n$-stars with $n>2$. The next results already solve the problem of computing the lower bounds of topological entropy of transitive star maps having the branching point fixed. 
The closures of the components of $X_{n} \backslash\{b\}$ will be called the branches of the $n$-star. Notice that each branch $B$ is a closed interval with endpoints $b$ and an endpoint $e$ of $X_{n}$. In what follows we will assume that the points of each branch $B$ are ordered as follows. If $x, y \in B, x \neq y$, we say that $x<y$ if $x$ is closer to $e$ than $y$. With this ordering on each branch we can write $B$ as the interval $[e, b]$.

Lemma 3.3. Let $f$ be a transitive $n$-star map with $n>2$ such that $f(b)=b$. Assume that there is a point $x$ such that $f(x)<x$. Then $h(f) \geq \log 2$.

Proof. Suppose that $x$ belongs to a branch $B=[e, b]$. Since $f(x) \in[e, x)$, there is a fixed point $y$ of $f$ in $[e, x)$. Therefore there are fixed points $c$ and $a$ in $B$ such that $c<x<a \leq b$ and there are no fixed points in $(c, a)$. Since $f(x)<x$, it follows that $f(t)<t$ for all $t \in(c, a)$. Hence, since $f([e, a])$ cannot be invariant by $f$ (otherwise $f$ would not be transitive), there exists $d \in[e, c)$ such that $f(d)=a$ and $f(z)<a$ for all $z \in(d, c)$. The interval $(d, a)$ is not invariant by $f$ (because again $f$ would not be transitive), and none of the points of $(d, a)$ is mapped to $a$. So there is $e \in(d, a)$ with $f(e)=d$. Now $f([d, e]) \cap f([e, a]) \supset[d, a]$. So $f$ is turbulent and hence $h(f) \geq \log 2$.

Let $f$ be an $n$-star map. A pair $(x, k) \in X_{n} \times \mathbb{N}$ will be called admissible if $f^{k}(x)<x$.

Lemma 3.4. Let $f$ be a transitive $n$-star map with $n>2$ such that $f(b)=b$. Assume that $f^{s}$ is transitive for all $s>1$. Then $h(f) \geq(\log 2) / n$.

Proof (Due to M. Misiurewicz). Because of transitivity of $f$, there are admissible pairs. Let $m$ be the minimum $k$ for which there exists some point $x$ such that $(x, k)$ is admissible. Then there is an admissible pair $(a, m)$. We claim that $m \leq n$.

Suppose that $m>n$. Then, among the points $a, f(a), f^{2}(a), \ldots, f^{m-1}(a)$ there are two points, say $f^{i}(a)$ and $f^{j}(a)$ which lie on the same branch. We may assume that $i<j$. By the definition of $m$, we have that $f^{i}(a)<f^{j}(a)$. Then we get a sequence of $m-(j-i)$ intervals

$$
[a, b],[f(a), b], \cdots,\left[f^{i-1}(a), b\right],\left[f^{j}(a), b\right],\left[f^{j+1}(a), b\right], \cdots,\left[f^{m-1}(a), b\right]
$$

with the property that the image of each interval contains the next interval of the sequence. Therefore there exists $a^{\prime} \in(a, b)$ such that $f^{m-(j-i)}\left(a^{\prime}\right)=f^{m}(a)<a<$ $a^{\prime}$. Since $m-(j-i)<m$ and the pair $\left(a^{\prime}, m-(j-i)\right)$ is admissible, we get a contradiction. This proves the claim.

Set $g=f^{m}$. Then $g$ is a transitive $n$-star map such that $g(b)=b$ and $g(a)<a$. By Lemma 3.3, $h(g) \geq \log 2$. So $h(f)=h\left(f^{m}\right) / m=h(g) / m \geq(\log 2) / m \geq$ $(\log 2) / n$.

Proposition 3.5. Let $f$ be a transitive $n$-star map with $n>2$ such that $f(b)=b$. Then $h(f) \geq(\log 2) / n$.

Proof. We start by defining recursively a sequence of length $N \leq n$ of pairs $\left(T^{i}, f_{i}\right)$ such that, for each $i \in\{1,2, \ldots, N\}, T^{i}$ is a union of $n_{i} \geq 1$ branches of $X_{n}$ and $f_{i}: T^{i} \longrightarrow T^{i}$ is continuous, transitive and verifies $f_{i}(b)=b$. First, set $T^{1}=X_{n}$ and $f_{1}=f$. Now assume that the pair $\left(T^{i}, f_{i}\right)$ has been defined for some $i \geq 1$. If either $n_{i}=1$ or $f_{i}^{s}$ is transitive for each $s \geq 1$, then we set $N=i$ and we end the procedure. Otherwise, by Proposition 3.1, $T^{i}$ splits into $k_{i} \geq 2$ nondegenerate closed subtrees $T_{1}^{i}, T_{2}^{i}, \ldots, T_{k_{i}}^{i}$ which intersect at a fixed point $y$ of $f_{i}$ and $f_{i}\left(T_{j}^{i}\right)=T_{j+1}^{i}$ for $j=1,2, \ldots, k_{i}-1$ and $f_{i}\left(T_{k_{i}}^{i}\right)=T_{1}^{i}$. Since $k_{i}>1$ and $f_{i}(b)=b$, 
we get that $y=b$. Take as $T^{i+1}$ the tree $T_{j_{i}}^{i}$ consisting of the smallest possible number of branches of $T^{i}$ (and hence of $X_{n}$ ) among all trees $T_{j}^{i}$ with $j=1,2, \ldots, k_{i}$. Then set $f_{i+1}=\left.f_{i}^{k_{i}}\right|_{T^{i+1}}$. Since $f_{i}$ was transitive on $T_{i}, f_{i+1}$ is transitive on $T^{i+1}$ by construction. This ends the definition of the pair $\left(T^{i+1}, f_{i+1}\right)$. We note that, by the minimality of the number of branches of $T_{j_{i}}^{i}$, we have that $n_{i+1} k_{i} \leq n_{i}$ for each $i$. Therefore, since $n_{1}=n$ we get inductively that $n_{i} \cdot \prod_{j=1}^{i-1} k_{j} \leq n$ for each $i$. Therefore, since $k_{i} \geq 2$ for each $i$, the above sequence is finite and $N \leq n$.

Now, clearly,

$$
h(f)=h\left(f_{1}\right) \geq \frac{1}{k_{1}} h\left(f_{2}\right) \geq \frac{1}{k_{1} k_{2}} h\left(f_{3}\right) \geq \ldots \geq \frac{1}{\prod_{i=1}^{N-1} k_{i}} h\left(f_{N}\right) .
$$

If $n_{N}=1$, then, since $f(b)=b$, by Theorem $3.2(\mathrm{a})$ we get that $f_{N}$ is turbulent. So, $h\left(f_{N}\right) \geq \log 2$ and, hence, $h(f) \geq(\log 2) /\left(\prod_{i=1}^{N-1} k_{i}\right) \geq(\log 2) / n$. Assume now that $n_{N}>1$. Then, by the definition of $N$ we have that $f_{N}^{s}$ is transitive for each $s \geq 1$. Thus, by Lemma 3.4 if $n_{N}>2$ or by Theorem 3.2 if $n_{N}=2$, we get that $h\left(f_{N}\right) \geq(\log 2) / n_{N}$. Hence, $h(f) \geq(\log 2) /\left(n_{N} \cdot \prod_{i=1}^{N-1} k_{i}\right) \geq(\log 2) / n$. This ends the proof of the proposition.

Now we will obtain lower bounds for the topological entropy of a transitive star map when the branching point is not fixed.

Let $f$ be an $n$-star map. We say that $x \in X_{n}$ is an upper (respectively down) crossing fixed point if $x$ is fixed, $x$ is neither $b$ nor an endpoint of $X_{n}$, and if $B$ is the branch containing $x$, then there exists $x_{1}<x$ such that $f(y) \leq y$ (respectively $f(y) \geq y)$ for all $y \in\left(x_{1}, x\right)$ and $\left.f\right|_{\left(x_{1}, x\right)} \neq \mathrm{Id}$.

We start by obtaining lower bounds of the topological entropy of transitive star maps with upper crossing fixed points.

Lemma 3.6. Let $f$ be a transitive $n$-star map with $n>2$. If $f$ has an upper crossing fixed point, then $h(f) \geq \log 2$.

Proof. Let $x$ be an upper crossing fixed point of $f$. Then $x$ is contained in some branch $B$ with endpoints $e$ and $b$. Let $y \in(e, x)$ be the largest point in that interval such that $f(y)=x$. Notice that $y$ exists; otherwise $f([e, x]) \subset[e, x]$ in contradiction with the transitivity of $f$. Clearly, since $x$ is an upper crossing fixed point, $f([y, x]) \subset[e, x]$.

There exists $z \in(y, x)$ such that $f(z)=y$; otherwise $f([y, x]) \subset[y, x]$ in contradiction with the transitivity of $f$. Then $f([y, z]) \cap f([z, x]) \supset[y, z] \cup[z, x]$. So $f$ is turbulent and hence $h(f) \geq \log 2$.

Proposition 3.7. Let $f$ be a transitive $n$-star map with $n>2$ such that $f(b) \neq b$. Then $h(f) \geq(\log 2) / 2$.

Proof. First suppose that $f^{2}$ is also transitive. Since $f(b) \neq b$, we have that $f(b) \in$ $B \backslash\{b\}$ for some branch $B=[e, b]$. Since $f(B) \not \subset B$ (otherwise $f$ would not be transitive) there exists $y \in B$ such that $f(y)=b$. We may assume that $y$ is the largest point with this property. Let $x$ be the infimum of the set of fixed points in $[y, b]$. Then, clearly, $x$ is a down crossing fixed point and $f([y, x]) \subset[y, b]$. On the other hand, $f([y, b]) \not \subset[y, b]$; otherwise $f$ is not transitive. Thus, there exists $z \in(x, b)$ such that $f(z)=y$ and $z$ is the smallest point having these properties. Therefore, since $f([y, x]) \supset[x, b]$, there exists $t \in(y, x)$ such that $f(t)=z$. Hence, $f^{2}(t)=y<t$ and $f^{2}(z)=b>z$. Consequently, $f^{2}$ has an upper crossing fixed 
point in $(t, z)$. Hence, since $f^{2}$ is transitive, by Lemma 3.6 we get that $h\left(f^{2}\right) \geq \log 2$. That is, $h(f) \geq(\log 2) / 2$.

Now assume that $f^{2}$ is not transitive. By Proposition 3.1 there exist a fixed point $y$ of $f$ of valence larger than or equal to $k>1$ and non-degenerate closed subtrees $T_{1}, \ldots, T_{k}$ of $T$ such that $T=\bigcup_{i=1}^{k} T_{i}, T_{i} \cap T_{j}=\{y\}$ for all $i \neq j, f\left(T_{i}\right)=T_{i+1}$ for $i=1, \ldots, k-1$ and $f\left(T_{k}\right)=T_{1}$.

Then, since $f(b) \neq b$ we get that $k=$ valence $(y)=2$. Then, without loss of generality, we may assume that $T_{1}$ is a closed interval. Clearly, $\left.f^{2}\right|_{T_{1}}: T_{1} \longrightarrow T_{1}$ is transitive. Therefore, since an endpoint of $T_{1}$ is a fixed point of $\left.f^{2}\right|_{T_{1}}$, by Theorem 3.2(a) we get that $\left.f^{2}\right|_{T_{1}}$ must be turbulent; so $h\left(\left.f^{2}\right|_{T_{1}}\right) \geq \log 2$. Hence $h(f)=h\left(f^{2}\right) / 2 \geq h\left(\left.f^{2}\right|_{T_{1}}\right) / 2 \geq(\log 2) / 2$.

Proof of Theorem 1.2. For $n=2$ the statement follows from Theorem 3.2. For $n>2$ we get the desired inequalities from Propositions 3.5 and 3.7.

To construct the transitive $n$-star map $g$ such that $g(b)=b$ and $h(g)=(\log 2) / n$ it is enough to take $g$ such that it maps cyclically each branch to the next one, all but one linearly, and the remaining one as in the tent map, piecewise linearly with two pieces. A simple computation shows that this map has the desired entropy.

Next we provide the promised example of a 3-star map $f$ such that $f(b) \neq b$ and $h(f)=\log 2 / 2$.

Example 3.8 (due to the referee). Let $X$ be a 3 -star and denote its endpoints by $a_{1}, a_{2}, a_{3}$. We choose four points $b>c_{1}>c_{2}>c_{3}>c_{4}>a_{3}$ and take a 3-star map $f$ such that $f\left(a_{1}\right)=a_{3}, f\left(a_{2}\right)=c_{1}, f\left(a_{3}\right)=a_{2}, f(b)=c_{3}, f\left(c_{1}\right)=c_{1}, f\left(c_{2}\right)=b$, $f\left(c_{3}\right)=a_{1}, f\left(c_{4}\right)=b$ and $f$ is injective (and continuous) on the closure of each connected component of $X \backslash\left\{b, c_{1}, c_{2}, c_{3}, c_{4}\right\}$. By using the arguments of the proof of Theorem 4.4.5 of [6] (by taking $P=\left\{b, a_{1}, a_{2}, a_{3}, c_{1}, c_{2}, c_{3}, c_{4}\right\}$ ) one can easily see that $h(f)=\log 2 / 2$.

\section{ENTROPY AND TRANSITIVE CIRCLE MAPS}

The goal of this section is to prove Theorem 1.3 and Proposition 1.4. To do it we will use the notation and definitions of [6]. We start by fixing the notation.

We consider the circle $\mathbb{S}^{1}$ as the set $\{z \in \mathbb{C}:|z|=1\}$. Let $f: \mathbb{S}^{1} \longrightarrow \mathbb{S}^{1}$ be a circle map. A continuous map $F: \mathbb{R} \longrightarrow \mathbb{R}$ such that $f \circ e=e \circ F$, where $e: \mathbb{R} \longrightarrow \mathbb{S}^{1}$ is the exponential map $e(X)=\exp (2 \pi i X)$, is called a lifting of $f$.

Let $a, b \in \mathbb{S}^{1}$. We will denote by $[a, b]$ the closed arc from $a$ to $b$ in $\mathbb{S}^{1}$. That is, the set $e([A, B])$ where $A \in e^{-1}(a)$ and $B \in e^{-1}(b) \cap[A, A+1)$. As usual, we will denote by $[a, b),(a, b]$ and $(a, b)$ the sets $[a, b] \backslash\{b\},[a, b] \backslash\{a\}$ and $[a, b] \backslash\{a, b\}$. Of course, $(a, b)$ is the interior of $[a, b]$ and will be called an open arc.

Now study the topological entropy of the transitive circle maps of degree 1 having periodic points to prove Proposition 1.4.

For $n \in \mathbb{N}$ we define $F_{n}: \mathbb{R} \longrightarrow \mathbb{R}$ as follows. For each $i \in \mathbb{Z}$, we set $F_{n}(X)=$ $X+1 / n$ if $X \in[i+1 / n, i+1], F_{n}(i+1 /(3 n))=i+2 / n, F_{n}(i+2 /(3 n))=i+1 / n$ and, on the intervals $[i, i+1 /(3 n)],[i+1 /(3 n), i+2 /(3 n)]$ and $[i+2 /(3 n), i+1 / n]$, $F_{n}$ is affine. Let $f_{n}$ be the circle map which has $F_{n}$ as a lifting. Clearly $f_{n}$ has degree 1.

Proof of Proposition 1.4. It is enough to show that the circle map $f_{n}$ of degree 1 is transitive, has periodic points and $h\left(f_{n}\right)=\log 3 / n$. To see it take $i \in$ 
$\mathbb{Z}$ and $l \in\{0,1, \ldots, n-1\}$ and set $I=e([i+l / n, i+(3 l+1) /(3 n)]), J=$ $e([i+(3 l+1) /(3 n), i+(3 l+2) /(3 n)]), K=e([i+(3 l+2) /(3 n), i+(l+1) / n])$. Clearly $f_{n}^{n}(I)=f_{n}^{n}(J)=f_{n}^{n}(K)=I \cup J \cup K$. So, the map $f_{n}^{n}$ has periodic points and, consequently $f_{n}$ has also periodic points. The map $\left.f_{n}^{n}\right|_{I \cup J \cup K}$ is transitive. Hence, so is $f_{n}$. Finally, from Lemma 4.1.10, Theorem 4.4.5 and the remarks at the beginning of Section 4.7 of [6] we see that $h\left(f_{n}^{n}\right)=\log 3$. So, $h\left(f_{n}\right)=\log 3 / n$.

Now we introduce the notation and preliminary results to prove Theorem 1.3.

A circle map $f$ has an $s$-horseshoe (respectively $s$-quasihorseshoe) with $s \geq 2$ if there are closed arcs (respectively subsets consisting of a finite number of closed arcs) $I_{i}$ of $\mathbb{S}^{1}$ for $i=1, \ldots, s$ such that their interiors are pairwise disjoint, and for all $i, j=1, \ldots, s$ there exists a closed arc (respectively a set which consists of a finite union of closed arcs) $\widetilde{I}_{i, j} \subset I_{i}$ such that $f\left(\widetilde{I}_{i, j}\right)=I_{j}$. From Proposition 4.3.2, Remark 4.3.4 and the comments at the beginning of Section 4.7 of [6] we get that if a circle map $f$ has an $s$-horseshoe (respectively an $s$-quasihorseshoe) with $s \geq 2$, then $h(f) \geq \log s$.

In the next two propositions we study the topological entropy for the transitive circle maps of degree 0 .

Proposition 4.1. Let $f: \mathbb{S}^{1} \longrightarrow \mathbb{S}^{1}$ be a transitive circle map of degree 0 . Then $h(f) \geq \log 2$.

Proof. Let $F: \mathbb{R} \longrightarrow \mathbb{R}$ be a lifting of $f$. Since $f$ has degree 0 , we have $F(X+1)=$ $F(X)$ for each $X \in \mathbb{R}$. Therefore $F(\mathbb{R})=F([0,1])$. Set $A=\min \{F(X): X \in[0,1]\}$ and set $B=\max \{F(X): X \in[0,1]\}$. Then $F(\mathbb{R})=[A, B]$. If $B<A+1$, then $f\left(\mathbb{S}^{1}\right) \neq \mathbb{S}^{1}$. So $f$ cannot be transitive. Consequently $B \geq A+1$. Let $U, V \in[A, A+1]$ be such that $F(U)=A$ and $F(V)=B$. If $u=e(U)$ and $v=e(V)$, then the two subintervals of $\mathbb{S}^{1}$ with endpoints $u$ and $v$ define a 2-quasihorseshoe for $f$. Hence $h(f) \geq \log 2$.

Proposition 4.2. There exists a transitive circle map $f: \mathbb{S}^{1} \longrightarrow \mathbb{S}^{1}$ of degree 0 such that $h(f)=\log 2$.

Proof. We define $F: \mathbb{R} \longrightarrow \mathbb{R}$ by $F(\mathbb{Z})=0, F(\mathbb{Z}+1 / 2)=1$, and $F$ is affine on each interval of the form $[i, i+1 / 2]$ and $[i+1 / 2, i+1]$ for each $i \in \mathbb{Z}$ (here $\mathbb{Z}+1 / 2$ denotes the set $\{i+1 / 2: i \in \mathbb{Z}\}$ ). Clearly, $F$ is a lifting of a circle map $f$ of degree 0. From Corollary 4.3.13 and the comments at the beginning of Section 4.6 of [6] we get that $h(f)=\log 2$.

In the rest of this section we will study the topological entropy for the transitive circle maps of degree -1 . We will start with some preliminary results.

Lemma 4.3. Let $f$ be a transitive circle map of degree -1 . Then exactly one of the following two statements holds.

(a) $f^{s}$ is transitive for all $s \geq 1$.

(b) There exist non-degenerate closed subintervals $J$ and $K$ of $\mathbb{S}^{1}$ such that $J \cup K=\mathbb{S}^{1}, J \cap K=\{y, z\}, y$ and $z$ are fixed points of $f, f(J)=K$ and $f(K)=J$. Moreover $\left.f^{2}\right|_{J}$ and $\left.f^{2}\right|_{K}$ are transitive.

Proof. First we must note that since the degree of $f$ is $-1, f$ has at least two fixed points (see for instance [6]). From Lemma 2.2 and Theorem 5.1 of [23], we get that either (a) holds or $f^{2}$ is not transitive. Now (b) follows using Proposition 3.1 with $s=2$. 
Lemma 4.4. Let $f$ be a circle map of degree -1 and let $F$ be a lifting of $f$. Then $F^{2}$ is the lifting of the circle map $f^{2}$ of degree 1 and the rotation interval $L_{F^{2}}$ is of the form $[-a, a]$ for some nonnegative real number $a$.

Proof. If $k / n \in L_{F^{2}}$, then, from Section 3.9 of [6], there exists $X \in \mathbb{R}$ such that $\left(F^{2}\right)^{n}(X)=X+k$. Then $\left(F^{2}\right)^{n}(F(X))=F\left(\left(F^{2}\right)^{n}(X)\right)=F(X+k)=F(X)-k$ because $f$ has degree -1 . Therefore the $F^{2}$-rotation number of $F(X)$ is $-k / n$. This ends the proof of the lemma.

The following result plays a key role in the study of the lower bounds of the topological entropy of transitive circle maps of degree -1 . The idea of the simple proof we present here is due to M. Misiurewicz.

Proposition 4.5. Let $f:[0,1] \longrightarrow[0,1]$ be a transitive interval map such that $f(0)=0$ and $f(1)=1$. Then $f$ has a 3 -horseshoe.

To prove Proposition 4.5 we shall use the following two lemmas. Consider the interval map $f:[0,1] \longrightarrow[0,1]$. We shall call a subinterval $[a, b]$ of $[0,1]$ a moving interval if $f(a)=a, f(b)=b$, and $f(t) \neq t$ for all $t \in(a, b)$. All interior points of a moving interval move in the same direction, that is either $f(t)<t$ for all $t \in(a, b)$ or $f(t)>t$ for all $t \in(a, b)$. Thus, we can speak about a left moving or right moving interval respectively.

Lemma 4.6. Let $f:[0,1] \longrightarrow[0,1]$ be a transitive interval map such that $f(0)=0$ and $f(1)=1$. Then $[0,1]$ is not a moving interval and $f$ has a fixed point in $(0,1)$ and a moving proper subinterval of $[0,1]$.

Proof. The fact that $[0,1]$ is not a moving interval follows immediately from the transitivity of $f$. Furthermore, if $f$ has no fixed points in $(0,1)$, then $[0,1]$ is moving; a contradiction. Since $f$ is transitive, there exists $x \in(0,1)$ such that $f(x) \neq x$. Clearly, $x$ belongs to a proper moving interval.

Lemma 4.7. Let $f:[0,1] \longrightarrow[0,1]$ be a transitive interval map such that $f(0)=0$ and $f(1)=1$.

(a) If $[a, b]$ is a right moving interval, then there exists $c \in[0,1]$ such that $c>b$ and $f(c) \leq a$.

(b) If $[a, b]$ is a left moving interval, then there exists $c \in[0,1]$ such that $c<a$ and $f(c) \geq b$.

Proof. We shall prove statement (a). Statement (b) follows in a similar way. Assume first that $a \neq 0$. By the transitivity of $f$, the subinterval $[a, 1]$ is not invariant. So there is $c \in[a, 1]$ such that $f(c)<a$. Since $f(t) \geq t \geq a$ for $t \in[a, b]$, we get that $c>b$.

Assume now that $a=0$. By Lemma 4.6 the interval $[0,1]$ is not moving and, hence, $b<1$. Let $d$ be the infimum of the set $f([b, 1])$. Assume that there is no $c>b$ with $f(c)=0$. If $d>0$, then the interval $[d, 1]$ is invariant which contradicts the transitivity of $f$. Consequently $d=0$ and there is $c>b$ such that $f(c)=0$.

Proof of Proposition 4.5. By Lemma 4.6 there exists a proper moving interval $[a, b]$. Assume that $[a, b]$ is right moving. If $[a, b]$ is left moving, then the proof follows similarly.

If $\max f([0, b])>\max f([a, b])$, then the point $d \in[0, b]$ at which $f(d)=$ $\max f([0, b])$ belongs to some right moving interval $\left[a^{\prime}, b^{\prime}\right] \neq[a, b]$. We can replace $[a, b]$ by $\left[a^{\prime}, b^{\prime}\right]$ and then we have $\max f\left(\left[0, b^{\prime}\right]\right)=\max f\left(\left[a^{\prime}, b^{\prime}\right]\right)$. Thus, we can 
assume that $\max f([0, b])=f(d)$ for some $d \in[a, b]$. We have that $f(d)>b$, since otherwise $[0, b]$ is invariant and, by transitivity, $b=1$ which is a contradiction with Lemma 4.7(a).

By Lemma 4.7(a), there is $c>b$ such that $f(c) \leq a$. This point $c$ belongs to some left moving interval $[p, q]$. We distinguish two cases.

Case 1. $q \leq f(d)$.

Then each of the intervals $[a, d],[d, c]$ and $[c, q] f$-covers $[a, q]$. So $f$ has a 3 horseshoe.

Case 2. $q>f(d)$.

By Lemma 4.7(b), there is a point $e<p$ such that $f(e) \geq q$. Since $f(e) \geq q>$ $f(d)=\max f([0, b])$, we get $e>b$. Then each of the intervals $[a, e],[e, c]$ and $[c, q]$ $f$-covers $[a, q]$. So $f$ has a 3 -horseshoe.

Now we are ready to prove our main result on transitive circle maps of degree -1 .

Proposition 4.8. Let $f: \mathbb{S}^{1} \longrightarrow \mathbb{S}^{1}$ be a transitive circle map of degree -1 . Then $h(f) \geq(\log 3) / 2$.

Proof. By Lemma 4.3 we can distinguish two cases.

Case 1. There exist $a$ and $b$, fixed points of $f$, such that $f([a, b])=[b, a], f([b, a])=$ $[a, b],\left.f^{2}\right|_{[a, b]}$ and $\left.f^{2}\right|_{[b, a]}$ are transitive.

Let $A \in e^{-1}(a)$ and $B \in e^{-1}(b) \cap[A, A+1)$. Clearly there exists a lifting $F$ of $f$ such that $F(A)=A$. Therefore, since $f^{2}(a)=a, f^{2}(b)=b, f^{2}([a, b])=[a, b]$, we get that $F^{2}(A)=A, F^{2}(B)=B$ and $F^{2}([A, B])=[A, B]$. Furthermore, since $\left.f^{2}\right|_{[a, b]}$ is transitive, so is $\left.F^{2}\right|_{[A, B]}$. Hence, from Proposition 4.5 it follows that $h\left(\left.F^{2}\right|_{[A, B]}\right) \geq \log 3$. Therefore, $h(f)=h\left(f^{2}\right) / 2 \geq h\left(\left.F^{2}\right|_{[A, B]}\right) / 2 \geq(\log 3) / 2$.

Case 2. $f^{s}$ is transitive for all $s \geq 1$.

Let $F$ be a lifting associated to $f$. First we assume that the rotation interval $L_{F^{2}}$ is non-degenerate. By Lemma 4.4, we know that 0 is an interior point of $L_{F^{2}}$. Then, from [36] it follows that $h\left(f^{2}\right) \geq \log 3$. Hence $h(f) \geq(\log 3) / 2$ and we are done.

Now we suppose that $L_{F^{2}}$ is degenerate. Again by Lemma 4.4 we obtain $L_{F^{2}}=$ $\{0\}$. Set $G=F^{2}$. Then $G$ is a lifting of the circle map $f^{2}$ of degree 1 . As usual we define the liftings $G_{l}$ and $G_{u}$ of degree 1 as follows: $G_{l}(X)=\inf \{G(Y): Y \geq X\}$, and $G_{u}(X)=\sup \{G(Y): Y \leq X\}$ (where $l$ stands for lower and $u$ for upper). Then $L_{G}=L_{G_{l}}=L_{G_{u}}=\{0\}$ (for more details see Section 3.7 of [6]). Moreover, it is well known that there exists $C \in \mathbb{R}$ such that $G(C)=G_{l}(C)=C$ (see for instance Theorem 3.7.20 of [6]). So for each $X \geq C$ we have $G(X) \geq G_{l}(X) \geq G_{l}(C)=C$. Similarly, there exists $D \in(C, C+1]$ such that $G(D)=G_{u}(D)=D$. Then, for each $X \in[C, D]$ we have $C \leq G(X) \leq G_{u}(X) \leq G_{u}(D)=D$. So $G(C)=C$, $G(D)=D$ and $G([C, D]) \subset[C, D]$. Therefore, $D=C+1$. Otherwise, the proper subinterval $e([C, D])$ of $\mathbb{S}^{1}$ is $f^{2}$-invariant, in contradiction with the transitivity of $f^{2}$. Hence, by Proposition $4.5,\left.G\right|_{[C, D]}$ has a 3 -horseshoe; and consequently $h\left(f^{2}\right) \geq h\left(\left.G\right|_{[C, D]}\right) \geq \log 3$. Thus $h(f)=h\left(f^{2}\right) / 2 \geq(\log 3) / 2$. 
Proposition 4.9. There exists a transitive circle map $f: \mathbb{S}^{1} \longrightarrow \mathbb{S}^{1}$ of degree -1 such that $h(f)=(\log 3) / 2$.

Proof. Let $g:[0,1] \longrightarrow[0,1]$ be the map such that $g(0)=1, g(1 / 2)=1 / 2, g(2 / 3)=$ $0, g(5 / 6)=1 / 2, g(1)=0$ and $g$ is affine on the intervals $[0,1 / 2],[1 / 2,2 / 3],[2 / 3,5 / 6]$ and $[5 / 6,1]$. Then, for each $X \in \mathbb{R}$ we define $F(X)=g(X-E(X))-E(X)$, where $E(\cdot)$ is the integer part function. Clearly, $F$ is a continuous map from $\mathbb{R}$ into $\mathbb{R}$ and $F(X+1)=F(X)-1$. So $F$ is a lifting of a circle map $f$ of degree -1 .

It is not difficult to see that the maps $\left.g^{2}\right|_{[0,1 / 2]}$ and $\left.g^{2}\right|_{[1 / 2,1]}$ are piecewise monotone in tree pieces with the absolute value of the slopes equal to 3 . Hence,

$$
h\left(\left.g^{2}\right|_{[0,1 / 2]}\right)=h\left(\left.g^{2}\right|_{[1 / 2,1]}\right)=\log 3
$$

and, consequently,

$$
h\left(g^{2}\right)=\max \left\{h\left(\left.g^{2}\right|_{[0,1 / 2]}\right), h\left(\left.g^{2}\right|_{[1 / 2,1]}\right)\right\}=\log 3
$$

(see for instance Lemma 4.1.10 of [6]). So,

$$
h(f)=h(F)=h(g)=h\left(g^{2}\right) / 2=(\log 3) / 2 .
$$

Proof of Theorem 1.3. Statements (b) and (c) follow from Propositions 4.1 and 4.8. The existence of the maps $f_{0}$ and $f_{-1}$ follows from Propositions 4.2 and 4.9. Lastly, it is easy to see that the map $f_{d}$ can be taken as the circle map which has $F(X)=d X$ as a lifting.

\section{Proof of Theorem 1.5}

Before going into the proof of Theorem 1.5 we fix some notation. For $f, \varphi \in \mathcal{C}(I)$ let $d_{1}(f, \varphi)$ denote $\max _{x \in I}|f(x)-\varphi(x)|$. If $(X, \rho)$ is any compact metric space and $f, \varphi \in \mathcal{C}(X)$, we use the same notation: $d_{1}(f, \varphi)=\max _{x \in X} \rho(f(x), \varphi(x))$. If $F(x, y)=\left(f(x), g_{x}(y)\right)$ and $\Phi(x, y)=\left(\varphi(x), \psi_{x}(y)\right)$ are maps from $\mathcal{C}_{\triangle}(X \times I)$ instead of the metric $d_{1}$, we will use the metric $d_{2}$ defined by

$$
\begin{aligned}
d_{2}(F, \Phi) & =\max _{(x, y) \in X \times I} \max \left\{\rho(f(x), \varphi(x)),\left|g_{x}(y)-\psi_{x}(y)\right|\right\} \\
& =\max \left\{d_{1}(f, \varphi), \max _{x \in X} d_{1}\left(g_{x}, \psi_{x}\right)\right\} .
\end{aligned}
$$

We note that the metric spaces $\left(\mathcal{C}(X), d_{1}\right)$ and $\left(\mathcal{C}_{\triangle}(X \times I), d_{2}\right)$ are complete and the convergence in them is uniform.

For $(x, y) \in X \times I$ let $\operatorname{pr}_{1}(x, y)=x$ and $\operatorname{pr}_{2}(x, y)=y$. The identity map on $I$ will be denoted by Id.

To prove Theorem 1.5 we need the following:

Lemma 5.1. Let $(X, \rho)$ be a compact metric space and let $F=\left(f, g_{x}\right)$ be a map from $\mathcal{C}_{\triangle}(X \times I)$ having all fiber maps nondecreasing and leaving the endpoints of $I$ fixed. Let $\left\{a_{1}, a_{2}, \ldots, a_{n}\right\}$ be a subset of $X$ and for $i=1,2, \ldots, n$ let $U_{i}$ be pairwise disjoint open sets such that $a_{i} \in U_{i}$. Suppose that $h_{i}$ are nondecreasing maps from $\mathcal{C}(I)$ leaving the endpoints of I fixed, with $d_{1}\left(h_{i}, g_{a_{i}}\right)<\varepsilon$ for some positive $\varepsilon$ and $i=1,2, \ldots, n$. Then there exists a map $\widetilde{F}=\left(f, \widetilde{g}_{x}\right) \in \mathcal{C}_{\triangle}(X \times I)$ having all fiber maps nondecreasing and leaving the endpoints of I fixed such that $d_{2}(F, \widetilde{F})<\varepsilon$, $\widetilde{g}_{a_{i}}=h_{i}$ for $i=1,2, \ldots, n$ and $\widetilde{g}_{x}=g_{x}$ for $x \in X \backslash \bigcup_{i=1}^{n} U_{i}$. 
Proof. For every $i=1,2, \ldots, n$ let $V_{i} \subset U_{i}$ be an open neighborhood of $a_{i}$ such that for some positive $\widetilde{\varepsilon}<\varepsilon, d_{1}\left(h_{i}, g_{x}\right)<\widetilde{\varepsilon}$ whenever $x \in V_{i}$.

We set $U=\bigcup_{i=1}^{n} U_{i}, V=\bigcup_{i=1}^{n} V_{i}$ and we choose $u: X \longrightarrow[0,1]$, a continuous function which takes the value 1 on $\left\{a_{1}, a_{2}, \ldots, a_{n}\right\}$ and 0 outside $V$. Replace every fiber map $g_{x}$ by $\widetilde{g}_{x}$, where

$$
\widetilde{g}_{x}(y)= \begin{cases}g_{x}(y) & \text { if } x \in X \backslash V \\ g_{x}(y)(1-u(x))+h_{i}(y) u(x) & \text { if } x \in V_{i}, i \in\{1,2, \ldots, n\},\end{cases}
$$

for every $y \in I$. Note also that for $x \in V_{i}$ and $i \in\{1,2, \ldots, n\}$ we can equivalently write

$$
\widetilde{g}_{x}(y)=u(x)\left(h_{i}(y)-g_{x}(y)\right)+g_{x}(y) .
$$

Take the map $\widetilde{F}=\left(f, \widetilde{g}_{x}\right)$. Clearly, $\widetilde{F} \in \mathcal{C}_{\triangle}(X \times I)$. From Equation (1) one can see that all fiber maps $\widetilde{g}_{x}$ are nondecreasing and leave the endpoints of $I$ fixed. Further, $\widetilde{g}_{a_{i}}=h_{i}$ for every $i$ and $\widetilde{g}_{x}=g_{x}$ for $x \in X \backslash V \supset X \backslash U$. Since for $x \in V_{i}$ and $i \in\{1,2, \ldots, n\}$ we have $d_{1}\left(h_{i}, g_{x}\right)<\widetilde{\varepsilon}$ and $u(x) \in[0,1]$, it follows from Equation (2) that $d_{1}\left(g_{x}, \widetilde{g}_{x}\right)<\widetilde{\varepsilon}$ whenever $x \in V$. Thus $d_{2}(F, \widetilde{F}) \leq \widetilde{\varepsilon}<\varepsilon$ and the proof is finished.

Proof of Theorem 1.5. Since $f$ is not minimal, there is a minimal set $M \neq X$. If the interior of $M$ is nonempty, then, since $M$ is invariant and $f$ is transitive, the set $M$ is dense in $X$. Since $M$ is closed, $M=X$; a contradiction. Therefore $M$ has empty interior and being closed, is nowhere dense.

Consider the set $\mathcal{F}$ of all maps $F=\left(f, g_{x}\right)$ from $\mathcal{C}_{\triangle}(X \times I)$ which satisfy the following three conditions:

(i) The basis map $f$ is the map from the assumptions of the theorem.

(ii) For every $x \in X$ the map $g_{x}$ is nondecreasing and leaves the endpoints of $I$ fixed.

(iii) $g_{x}$ is the identity map for all $x \in M$.

Every map in $\mathcal{F}$, having all fiber maps monotone, has the same entropy as $f$ (see Section 1). So it suffices to show that there is a transitive map in $\mathcal{F}$. We are going to prove it by the category method. The category arguments will work since $\mathcal{F}$, being a non-empty closed subset of the space $\mathcal{C}_{\triangle}(X \times I)$, is a complete metric space.

Let $\left\{U_{i}^{X}\right\}_{i=1}^{\infty}$ be a basis for the topology of $X$ and let $\left\{U_{i}^{I}\right\}_{i=1}^{\infty}$ be all open intervals with rational endpoints lying in the open interval $(0,1)$. Let $\left\{U_{i}\right\}_{i=1}^{\infty}$ be an enumeration of the set $\left\{U_{i}^{X} \times U_{j}^{I}: i, j \in \mathbb{N}\right\}$. Then any open ball in $X \times I$ contains some of the open sets $U_{i}$. For any positive integers $i, j$ let

$$
T_{i j}=\left\{G \in \mathcal{F}: G^{k}\left(U_{i}\right) \cap U_{j} \neq \emptyset \text { for some positive integer } k\right\} .
$$

Then, a map from $\mathcal{F}$ is transitive if and only if it belongs to $\bigcap_{i, j} T_{i j}$.

For any $i, j$ the set $T_{i j}$ is open in $\mathcal{F}$. Suppose we have proved that the sets $T_{i j}$ are dense in $\mathcal{F}$. Then, by Baire Category Theorem, $\bigcap_{i, j} T_{i j}$ is a non-empty set (in fact, residual in $\mathcal{F}$ ). Thus there is a transitive map in $\mathcal{F}$.

So the only thing which remains to prove is that $T_{i j}$ is dense in $\mathcal{F}$ for every $i, j$. To this end fix some $i, j$ and take any $F=\left(f, g_{x}\right) \in \mathcal{F}$ and $\varepsilon>0$. We are going to show that $d_{2}(G, F)<\varepsilon$ for some $G \in T_{i j}$.

First, take a positive integer $N \geq 4 / \varepsilon$. Further, take an open neighborhood $V$ of the set $M$ in the space $(X, \rho)$ such that $d_{1}\left(g_{x}\right.$, Id) $<\varepsilon / 4$ for every $x \in V$ (note that 
$g_{x}=\mathrm{Id}$ for $\left.x \in M\right)$. Now let $x_{0} \in \operatorname{pr}_{1}\left(U_{i}\right)$ be a point with dense $f$-orbit. Since $M$ is invariant and nowhere dense, the orbit of $x_{0}$ is disjoint with $M$ and there are nonnegative integers $r, s$ such that $f^{r}\left(x_{0}\right), f^{r+1}\left(x_{0}\right), \ldots, f^{r+N-1}\left(x_{0}\right)$ belong to $V$ and $f^{r+N+s}\left(x_{0}\right) \in \operatorname{pr}_{1}\left(U_{j}\right)$. Since all the fiber maps of $F$ are onto, there is a point $y^{*} \in(0,1)$ such that $\left(f^{r+N}\left(x_{0}\right), y^{*}\right)$ is mapped by $F^{s}$ into the intersection of the fiber $I\left(f^{r+N+s}\left(x_{0}\right)\right)$ with $U_{j}$. Now take any $y_{0}$ with $\left(x_{0}, y_{0}\right) \in U_{i}$.

Case 1. $z=\operatorname{pr}_{2}\left(F^{r}\left(x_{0}, y_{0}\right)\right)$ is neither 0 nor 1 .

Consider a map $g \in \mathcal{C}(I)$ having the following three properties:

(i) $d_{1}(g$, Id $)<\varepsilon / 4$,

(ii) $g$ is a nondecreasing map leaving the endpoints of $I$ fixed,

(iii) $g^{N}(z)=y^{*}$.

Clearly, such a map $g$ exists. Indeed, if $y^{*}=z$, take $g=$ Id. If $y^{*}>z$, take $g$ such that $g(0)=0, g(1)=1, g(z)=z+\left(y^{*}-z\right) / N, g\left(y^{*}-\left(y^{*}-z\right) / N\right)=y^{*}$ and $g$ is linear on the intervals $[0, z],\left[z, y^{*}-\left(y^{*}-z\right) / N\right]$ and $\left[y^{*}-\left(y^{*}-z\right) / N, 1\right]$. If $y^{*}<z$, then we define $g$ similarly.

By Lemma 5.1 we can take a map $G=\left(f, \widetilde{g}_{x}\right) \in \mathcal{F}$ such that $d_{2}(G, F)<\varepsilon / 2$ and

$$
\widetilde{g}_{x}= \begin{cases}g_{x} & \text { if } x \in\left\{f^{k}\left(x_{0}\right): 0 \leq k \leq r-1 \text { or } r+N \leq k \leq r+N+s-1\right\} \\ g & \text { if } x \in\left\{f^{k}\left(x_{0}\right): r \leq k \leq r+N-1\right\} .\end{cases}
$$

We note that, for $x \in\left\{f^{r}\left(x_{0}\right), f^{r+1}\left(x_{0}\right), \ldots, f^{r+N-1}\left(x_{0}\right)\right\}$, we have

$$
d_{1}\left(\widetilde{g}_{x}, g_{x}\right)=d_{1}\left(g, g_{x}\right) \leq d_{1}(g, \mathrm{Id})+d_{1}\left(g_{x}, \mathrm{Id}\right)<\varepsilon / 4+\varepsilon / 4=\varepsilon / 2 .
$$

Since

$$
\begin{aligned}
G^{r+N+s}\left(x_{0}, y_{0}\right) & =G^{N+s}\left(F^{r}\left(x_{0}, y_{0}\right)\right)=G^{N+s}\left(f^{r}\left(x_{0}\right), z\right) \\
& =G^{s}\left(f^{r+N}\left(x_{0}\right), y^{*}\right)=F^{s}\left(f^{r+N}\left(x_{0}\right), y^{*}\right) \in U_{j},
\end{aligned}
$$

we have $G \in T_{i j}$. So, in Case 1 we are done.

Case 2. $\operatorname{pr}_{2}\left(F^{r}\left(x_{0}, y_{0}\right)\right)$ is either 0 or 1 .

Then we use Lemma 5.1 to get a map $H=\left(f, h_{x}\right) \in \mathcal{F}$ such that $d_{2}(H, F)<\varepsilon / 2$ and the fiber maps $h_{x}$ are strictly increasing for $x \in\left\{x_{0}, f\left(x_{0}\right), \ldots, f^{r-1}\left(x_{0}\right)\right\}$. Since $y_{0}$ is neither 0 nor 1 , we have that $\operatorname{pr}_{2}\left(H^{r}\left(x_{0}, y_{0}\right)\right)$ is neither 0 nor 1 . So, using the same argument as in Case 1 , we can find a map $G \in T_{i j}$ such that $d_{2}(G, H)<\varepsilon / 2$. Then $d_{2}(G, F)<\varepsilon$ and the theorem is proved.

\section{Proof of Theorem 1.7}

In the proof of Theorem 1.7 the tent map $f(x)=1-|2 x-1|$ from $I$ to itself will play a key role. So, before going into the proof of this theorem, we will recall some of the basic properties of $f$.

(T.1) $f$ is transitive.

(T.2) $f$ has a dense set of periodic points and each of them is a rational number.

(T.3) The set of all preimages of $1 / 2$ is dense in $I$.

(T.4) The points 0 and $2 / 3$ are the only fixed points of $f$.

(T.5) $h(f)=\log 2$. 
Set $L=[0,1 / 2]$ and $R=[1 / 2,1]$. We will say that $A_{0} \ldots A_{n-1}$, where $A_{i} \in\{L, R\}$, is the itinerary of length $n$ of a point $x \in I$ if $f^{i}(x) \in A_{i}$ for $i=0, \ldots, n-1$. Note that if $f^{j}(x)=1 / 2$ for some $j<n$, then $x$ has two distinct itineraries of length $n$. We will use the following two additional simple properties of itineraries.

(T.6) If $x$ and $y$ have the same itinerary of length $n$, then $|x-y| \leq 2^{-n}$.

(T.7) For a given sequence $\underline{\mathbf{K}}$ of length $n$ of $L$ 's and $R$ 's and $x \in I$ there exists $y \in I$ with $\underline{\mathbf{K}}$ as the itinerary of length $n$ of $y$ and such that $f^{n}(y)=x$.

We will use notation $\underline{\mathbf{K M}}$ for the concatenation of itineraries $\underline{\mathbf{K}}$ and $\underline{\mathbf{M}}$ and $\underline{\mathbf{K}}^{n}$ for the concatenation of $n$ copies of $\underline{\mathbf{K}}$.

Proof of Theorem 1.7. Fix an irrational number $\beta \in(0,2 / 3)$. Since all fiber maps $g_{x}(y)=y^{\exp (x-\beta)}$ are monotone, from Bowen's formula (see Section 1) we get $h(F)=h(f)=\log 2$.

Now we prove that the set of periodic points of $F$ is contained in $I \times\{0,1\}$. For $x \in I$ and $n>0$ we define

$$
S_{n}(x)=\sum_{i=0}^{n-1} f^{i}(x) .
$$

Then the fiber map of $F^{n}$ at the fiber having basis $x$ is given by

$$
g_{f^{n-1}(x)} \circ \ldots \circ g_{f(x)} \circ g_{x}(y)=y^{\exp \left(S_{n}(x)-n \beta\right)} .
$$

If $F^{n}(x, y)=(x, y)$, then $y^{\exp \left(S_{n}(x)-n \beta\right)}=y$ and $f^{i}(x)$ is periodic for $f$ for every $i$. Since periodic points of $f$ are rational and $\beta$ is irrational, $S_{n}(x)-n \beta \neq 0$. Therefore $y \in\{0,1\}$.

Now only it remains to prove that $F$ is transitive. Note that it is enough to show that for each pair of points $\left(x_{0}, y_{0}\right),\left(x_{1}, y_{1}\right) \in I^{2}$ and each $\varepsilon>0$ there is a point $\left(x_{2}, y_{2}\right) \in I^{2}$ and $n>0$ such that $\left|x_{2}-x_{0}\right|<\varepsilon,\left|y_{2}-y_{0}\right|<\varepsilon,\left|f^{n}\left(x_{2}\right)-x_{1}\right|<\varepsilon$ and $\left|y_{2}^{\exp \left(S_{n}\left(x_{2}\right)-n \beta\right)}-y_{1}\right|<\varepsilon$.

In what follows we will show the existence of the point $\left(x_{2}, y_{2}\right)$. By (T.3) there exists a point $t \in I$ such that $\left|t-x_{0}\right|<\varepsilon / 2$ and $f^{m}(t)=1 / 2$ for some $m>0$. Let $\underline{\mathbf{K}}$ be the itinerary of $t$ of length $m$. From (T.7), for every $k, l>0$ there is a point $a_{k, l} \in I$ with the itinerary of length $n=m+2+k+l$ equal to $\underline{\mathbf{K}} R^{2} L^{k} R^{l}$ and such that $f^{n}\left(a_{k, l}\right)=x_{1}$. Since, for $i=0, \ldots, m+1$, the itineraries of length $m+2+k-i$ of the points $f^{i}(t)$ and $f^{i}\left(a_{k, l}\right)$ coincide, by (T.6) we have

$$
\left|f^{i}(t)-f^{i}\left(a_{k, l}\right)\right| \leq 2^{i-m-2-k} .
$$

On the other hand, for $i=0, \ldots, k-1$, the itineraries of length $k+l-i$ of the points $2^{i-k}(2 / 3)$ and $f^{m+2+i}\left(a_{k, l}\right)$ also coincide. So, again by (T.6), we have

$$
\left|2^{i-k}(2 / 3)-f^{m+2+i}\left(a_{k, l}\right)\right| \leq 2^{i-k-l} .
$$

Moreover, the point $b=f^{m+2+k}\left(a_{k, l}\right)$ has itinerary $R^{l}$ and $f^{l}(b)=x_{1}$, so $b=$ $2 / 3+\left(x_{1}-2 / 3\right)(-2)^{-l}$. Therefore, for $i=0, \ldots, l-1$, we have

$$
f^{m+2+k+i}\left(a_{k, l}\right)=2 / 3+\left(x_{1}-2 / 3\right)(-2)^{i-l} .
$$

Putting together our estimates, we get

$$
\left|S_{n}\left(a_{k, l}\right)-\left(S_{m+2}(t)+C_{0}\right)\right| \leq \sum_{i=0}^{m+1} 2^{i-m-2-k}+\sum_{i=0}^{k-1} 2^{i-k-l},
$$


where

$$
\begin{aligned}
C_{0} & =\frac{2}{3} \sum_{i=0}^{k-1} 2^{i-k}+\frac{2}{3} l+\left(x_{1}-2 / 3\right) \sum_{i=0}^{l-1}(-2)^{i-l} \\
& =\frac{2}{3}\left(1-2^{-k}\right)+\frac{2}{3} l+\left(x_{1}-2 / 3\right) \frac{1}{3}\left(-1+(-2)^{-l}\right) .
\end{aligned}
$$

Therefore, if we set $C_{1}=S_{m+2}(t)+2 / 3-\left(x_{1}-2 / 3\right) / 3$, we get

$$
\begin{aligned}
& \left|S_{n}\left(a_{k, l}\right)-C_{1}-\frac{2}{3} l\right|-\left|\frac{2}{3} 2^{-k}-\left(x_{1}-2 / 3\right) \frac{1}{3}(-2)^{-l}\right| \\
& \quad \leq\left|S_{n}\left(a_{k, l}\right)-\left(S_{m+2}(t)+C_{0}\right)\right| \leq\left(2^{-k}-2^{-(m+2+k)}\right)+\left(2^{-l}-2^{-(k+l)}\right) .
\end{aligned}
$$

Hence, since $\left|x_{1}-2 / 3\right|<1$, we have

$$
\begin{aligned}
& \left|S_{n}\left(a_{k, l}\right)-C_{1}-\frac{2}{3} l\right| \\
& \quad \leq\left(\frac{2}{3} 2^{-k}+2^{-k}-2^{-(m+2+k)}\right)+\left(\frac{1}{3} 2^{-l}+2^{-l}-2^{-(k+l)}\right) \\
& \quad \leq 2^{-k+1}+2^{-l+1} .
\end{aligned}
$$

Note that from Equation (3) we obtain $\left|t-a_{k, l}\right| \leq 2^{-m-2-k}$. So, there exists $k^{*}$ such that $\left|a_{k, l}-x_{0}\right|<\varepsilon$ for every $k>k^{*}$. Since $f^{n}\left(a_{k, l}\right)=x_{1}$, if we take $x_{2} \in\left\{a_{k, l}: k>k^{*}\right.$ and $\left.l>0\right\}$, it will remain only to show that we can choose $y_{2}$ such that $\left|y_{2}-y_{0}\right|<\varepsilon$ and

$$
\left|y_{2}^{\exp \left(S_{n}\left(a_{k, l}\right)-n \beta\right)}-y_{1}\right|<\varepsilon .
$$

We choose as $y_{2}$ any point different from zero and one such that $\left|y_{2}-y_{0}\right|<\varepsilon$. Note that, without loss of generality, $y_{1}$ may also be assumed to be different from zero and one. So, there is a unique real number $w$ such that $y_{2}^{\exp (w)}-y_{1}=0$. So there is some $\delta>0$ such that if $\left|S_{n}\left(a_{k, l}\right)-n \beta-w\right|<\delta$, then Equation (5) is satisfied. In short, we have to show that given $w \in \mathbb{R}$ and $\delta>0$ there exist $k>k^{*}$ and $l$ such that $\left|S_{n}\left(a_{k, l}\right)-n \beta-w\right|<\delta$.

To this end we rewrite Equation (4) as

$$
\left|\left[S_{n}\left(a_{k, l}\right)-n \beta-w\right]-\left[C_{2}-k \beta+l(2 / 3-\beta)\right]\right| \leq 2^{-k+1}+2^{-l+1},
$$

where $C_{2}=C_{1}-w-(m+2) \beta$. Let $k^{* *} \geq k^{*}$ and $l^{*}$ be such that $2^{-k^{* *}+1}+2^{-l^{*}+1}<$ $\delta / 2$. So, our problem is reduced to showing that there exist $k>k^{* *}$ and $l>l^{*}$ such that

$$
\left|C_{2}-k \beta+l(2 / 3-\beta)\right|<\delta / 2 .
$$

Since $\beta>0$, Equation (6) is is equivalent to showing that there are $k>k^{* *}$ and $l>l^{*}$ large enough such that

$$
\left|C_{3}-k+l \gamma\right|<\frac{\delta}{2 \beta},
$$

where $C_{3}=C_{2} / \beta$ and $\gamma=\frac{2}{3 \beta}-1$. Since $\beta$ is irrational, so is $\gamma$. Moreover, $0<\beta<2 / 3$ and so $\gamma$ is positive. Therefore, Equation (7) holds for some $k>k^{* *}$ and $l>l^{*}$ because each orbit of an irrational rotation of the circle is dense. Hence, the theorem follows by taking $x_{2}=a_{k, l}$. 


\section{Proof of Theorem 1.9}

(a) Let $F=\left(f, g_{x}\right) \in \mathcal{C}_{\triangle}(X \times I)$ be transitive. Then so is $f \in \mathcal{C}(X)$. As it was said in the introduction, $h(F) \geq h(f)$ and we get one inequality. The converse inequality and the rest of the statement follow from Theorem 1.5.

(b) It follows from Theorem 3.2 and (a) by using induction on $n$.

(c) First consider the case $n=2$. Take a transitive self-map of the unit interval $I$ having finite entropy and leaving the endpoints 0 and 1 fixed, say the map $f(x)=$ $|1-| 3 x-1||$ with $h(f)=\log 3$. Slightly modifying the proof of Theorem 1.5 (additionally demanding that in the definition of the set $\mathcal{F}$ also $g_{0}$ and $g_{1}$ be the identity maps) one can show that $f$ can be extended to a map $F=\left(f, g_{x}\right) \in \mathcal{C}_{\triangle}\left(I^{2}\right)$ such that $F$ is transitive, $h(F)=h(f)$, all fiber maps of $F$ are nondecreasing and leave the endpoints of $I$ fixed, and $g(0, y)=g(1, y)=y$ for every $y \in I$.

Now let $m$ be any positive integer. Consider the unit disk $D^{2}$ and its sector $A$ given in polar coordinates by $A=\left\{(\varphi, \varrho) \in D^{2}: \varphi \in\left[0, \frac{2 \pi}{m}\right]\right\}$. Define

$$
\psi_{1}(\varphi, \varrho)= \begin{cases}\left(\frac{2 \pi}{m} f\left(\frac{m}{2 \pi} \varphi\right), g\left(\frac{m}{2 \pi} \varphi, \varrho\right)\right), & \text { if }(\varphi, \varrho) \in A \\ (\varphi, \varrho), & \text { if }(\varphi, \varrho) \in D^{2} \backslash A .\end{cases}
$$

It is easy to see that $\psi_{1}$ is a continuous map from $D^{2}$ onto itself. Since $F=\left(f, g_{x}\right)$ is transitive, then so is $\left.\psi_{1}\right|_{A}$. Further, $h\left(\left.\psi_{1}\right|_{A}\right)=h\left(\psi_{1}\right)=\log 3$. In fact, $\left.\psi_{1}\right|_{A}$ is topologically semiconjugate to $F$ and so $h\left(\left.\psi_{1}\right|_{A}\right) \leq h(F)=\log 3$. On the other hand, $\psi_{1} \mid B$ where $B=\{(\varphi, \varrho) \in A: \varrho=1\}$, is topologically conjugate to $f$ and so $h\left(\left.\psi_{1}\right|_{A}\right) \geq h\left(\psi_{1} \mid B\right)=\log 3$.

Now take the rotation $\psi_{2}(\varphi, \varrho)=\left(\varphi+\frac{2 \pi}{m}, \varrho\right)$ and define $\psi=\psi_{2} \circ \psi_{1}$. Then $\psi$ is a continuous map from $D^{2}$ onto itself. Since $\left.\psi_{1}\right|_{A}$ is transitive, it is easy to see that $\psi$ is transitive. Moreover the restrictions of $\psi^{m}$ to the sets $\psi_{2}^{i}(A), i=$ $0,1, \ldots, m-1$ are topologically conjugate. Thus $h\left(\psi^{m}\right)=h\left(\left.\psi^{m}\right|_{A}\right)=h\left(\left.\psi_{1}\right|_{A}\right)=$ $\log 3$. Consequently $h(\psi)=\frac{1}{m} \log 3$.

Let $H$ be a homeomorphism of the square $I^{2}$ onto the disk $D^{2}$ and $\Psi=H^{-1}$ 。 $\psi \circ H$. Then $\Psi$ is a transitive self-map of $I^{2}$ with $h(\Psi)=h(\psi)=\frac{1}{m} \log 3$. Since $m$ was an arbitrary positive integer, the proof is finished in the case $n=2$.

For $n>2$ use Theorem 1.5 and induction on $n$.

(d) We prove this for the unit disk $D^{2}$. Then use the fact that $I^{2}$ and $D^{2}$ are homeomorphic. As the desired map we take the Shnirelman-Besicovitch-Anosov map from $D^{2}$ onto itself given in polar coordinates by ([40], [41])

$$
H(\varphi, \varrho)= \begin{cases}(\varphi+\theta, \varrho) & \text { if } \varrho \in\{0,1\}, \\ \left(\varphi+\theta, g\left[g^{-1}(\varrho)+f(\varphi)\right]\right) & \text { if } \varrho \in(0,1),\end{cases}
$$

where $g(x)=1 / \ln \left[e-x+\left(x^{2}+1\right)^{\frac{1}{2}}\right], \theta$ is incommensurable with $2 \pi$ and $f$ is a continuous $2 \pi$-periodic function. Here we assume that $\theta$ and $f$ are such that $H$ is transitive (Sidorov in [40] proved that such $\theta$ and $f$ exist). It is easy to see that $H$ is an orientation preserving homeomorphism of $D^{2}$. Moreover, $H$ is strictly increasing with respect to the variable $\varrho$ in the following sense: For each $\varrho_{1}<\varrho_{2}$ and $\varphi \in[0,2 \pi]$ we have that $p_{2}\left(H\left(\varphi, \varrho_{1}\right)\right)<p_{2}\left(H\left(\varphi, \varrho_{2}\right)\right)$ where $p_{2}(\varphi, \varrho)=\varrho$.

Consider the sets $D^{2}, D_{0}^{2}=D^{2} \backslash\{0,0\}, A^{2}=\{(\varphi, \varrho): 1 \leq \varrho \leq 2\}$ and $A_{0}^{2}=$ $A^{2} \backslash\{(\varphi, \varrho): \varrho=1\}$. We define the maps $\psi: D_{0}^{2} \longrightarrow A_{0}^{2}$ by $\psi(\varphi, \varrho)=(\varphi, \varrho+1)$, $H_{0}=\left.H\right|_{D_{0}^{2}}: D_{0}^{2} \longrightarrow D_{0}^{2}$, and $G_{0}=\psi \circ H_{0} \circ \psi^{-1}: A_{0}^{2} \longrightarrow A_{0}^{2}$. Finally we define 
$G: A \longrightarrow A$ by

$$
G(\varphi, \varrho)= \begin{cases}G_{0}(\varphi, \varrho) & \text { if } \varrho>1 \\ (\varphi+\theta, \varrho) & \text { if } \varrho=1\end{cases}
$$

Now take the following four quantities:

$$
\begin{aligned}
& h_{1}=h(H)=\lim _{\varepsilon \rightarrow 0} \limsup _{n \rightarrow \infty} \frac{1}{n} \log s_{n}\left(\varepsilon, D^{2}, H\right), \\
& h_{2}=\lim _{\varepsilon \rightarrow 0} \limsup _{n \rightarrow \infty} \frac{1}{n} \log s_{n}\left(\varepsilon, D_{0}^{2}, H_{0}\right), \\
& h_{3}=\lim _{\varepsilon \rightarrow 0} \limsup _{n \rightarrow \infty} \frac{1}{n} \log s_{n}\left(\varepsilon, A_{0}^{2}, G_{0}\right), \text { and } \\
& h_{4}=h(G)=\lim _{\varepsilon \rightarrow 0} \limsup _{n \rightarrow \infty} \frac{1}{n} \log s_{n}\left(\varepsilon, A^{2}, G\right) .
\end{aligned}
$$

(Despite the fact that the sets $D_{0}^{2}$ and $A_{0}^{2}$ are not compact, $s_{n}\left(\varepsilon, D_{0}^{2}, H_{0}\right)$ and $s_{n}\left(\varepsilon, A_{0}^{2}, G_{0}\right)$ are finite numbers because $H_{0}$ and $G_{0}$ are restrictions of $H$ and $G$.)

We have $h_{1}=h_{2}$ which is an easy consequence of the fact that $D_{0}^{2}$ differs from $D^{2}$ only in one point. Further, $h_{2} \leq h_{3}$ since for any $x, y \in D_{0}^{2}, \mathrm{~d}(x, y) \leq \mathrm{d}(\psi(x), \psi(y))$. Trivially $h_{3} \leq h_{4}$ since $G_{0}=\left.G\right|_{A_{0}^{2}}$. Finally, if we define a map

$$
\pi: A^{2} \longrightarrow Y=\{(\varphi, \varrho): \varrho=2\}
$$

by $\pi(\varphi, \varrho)=(\varphi, 2)$ and $S: Y \longrightarrow Y$ by $S(\varphi, 2)=(\varphi+\theta, 2)$, then Bowen's formula (see Theorem 17 in [21]) gives

$$
h_{4} \leq h(S)+\sup _{y \in Y} h\left(G, \pi^{-1}(y)\right)=0 .
$$

To prove that $\sup _{y \in Y} h\left(G, \pi^{-1}(y)\right)=0$ we use that $G$ is strictly increasing with respect to the variable $\varrho$ and the fact that any sequence of monotone maps on the interval has zero topological entropy ([32], cf. also the discussion on the Bowen's formula and the topological entropy of triangular maps in the introduction of this paper).

Thus we have proved $0 \leq h_{1}=h_{2} \leq h_{3} \leq h_{4} \leq 0$ and so $h_{1}=0$.

Now (e) follows from (d) and (a).

\section{REFERENCES}

[1] R. Adler, A. Konheim and J. McAndrew, Topological entropy, Trans. Amer. Math. Soc. 114 (1965), 309-319. MR 30:5291

[2] Ll. Alsedà, S. F. Kolyada and L. Snoha, On topological entropy of triangular maps of the square, Bull. Austral. Math. Soc. 48 (1993), 55-67. MR 94d:54087

[3] Ll. Alsedà, M. A. del Río and J. A. Rodríguez, Lower bounds of the topological entropy for transitive graph maps, preprint, 1996.

[4] Ll. Alsedà and J. Llibre, Periods for triangular maps, Bull. Austral. Math. Soc. 47 (1993), 41-53. MR 93m:58033

[5] Ll. Alsedà, J. Llibre and M. Misiurewicz, Periodic orbits of maps of $Y$, Trans. Amer. Math. Soc. 313 (1989), 475-538. MR 90c:58145

[6] Ll. Alsedà, J. Llibre and M. Misiurewicz, Combinatorial dynamics and entropy in dimension one, Advanced Series in Nonlinear Dynamics, Vol. 5, World Scientific, Singapore, 1993. MR 95j:58042

[7] Ll. Alsedà, S. Baldwin, J. Llibre and M. Misiurewicz, Entropy of transitive tree maps, Topology 36 (1996), 519-532. MR 98f:54031

[8] Ll. Alsedà and X. Ye, Division for star maps with the central point fixed, Acta Mathematica Universitatis Comenianae LXII(2) (1993), 237-248. MR 95e:58057 
[9] D. V. Anosov and A. B. Katok, New examples in smooth ergodic theory. Ergodic diffeomorphisms, Trans. Moscow. Math. Soc. 23 (1970), 1-35. MR 51:6888

[10] J. Auslander and Y. Katznelson, Continuous maps of the circle without periodic points, Israel J. Math. 32 (1979), 375-381. MR 81e:58048

[11] S. Baldwin, An extension of Šarkovskii's Theorem to the $n$-od, Ergod. Th. \& Dynam. Sys. 11 (1991), 249-271. MR 91h:58159

[12] S. Baldwin and J. Llibre, Periods of maps on trees with all branching points fixed, Ergod. Th. \& Dynam. Sys. 15 (1995), 239-246. MR 96e:58126

[13] M. Barge and J. Martin, Chaos, periodicity, and snakeline continua, Trans. Amer. Math. Soc. 289 (1985), 355-365. MR 86h:58079

[14] M. Barge and J. Martin, Dense periodicity on the interval, Proc. Amer. Math. Soc. 94 (1985), 731-735. MR 87b:58068

[15] L. Block and A. Coppel, Dynamics in One Dimension, Lecture Notes in Math. 1513, Springer, Berlin, 1992. MR 93g:58091

[16] L. Block and E. M. Coven, Topological conjugacy and transitivity for a class of piecewise monotone maps of the interval, Trans. Amer. Math. Soc. 300 (1987), 297-306. MR 88c:58032

[17] L. Block, J. Guckenheimer, M. Misiurewicz and L. S. Young, Periodic points and topological entropy of one-dimensional maps, Lecture Notes in Math. 819, pp. 18-34, Springer, Berlin, 1980. MR 82j:58097

[18] A. Blokh, On sensitive mappings of the interval, Russian Math. Surveys 37:2 (1982), 189-190. MR 89k:58053

[19] A. M. Blokh, On transitive mappings of one-dimensional branched manifolds (in Russian), Diff. -Difference Equations and Problems of Mathematical Physics, Inst. of Math., Kiev (1984), 3-9. MR 88b:58111

[20] A. Blokh, On the connection between entropy and transitivity for one-dimensional mappings, Russian Math. Surveys 42:5 (1987), 209-210. MR 89g:58117

[21] R. Bowen, Entropy for group endomorphisms and homogeneous spaces, Trans. Amer. Math. Soc. 153 (1971), 401-414. MR 43:469

[22] P. Collet and J. P. Eckmann, Iterated maps on the interval as dynamical systems, Progress in Phys. 1, Birkhäuser, Boston, 1980. MR 82j:58078

[23] E. M. Coven and I. Mulvey, Transitivity and the centre for maps of the circle, Ergod. Th. \& Dynam. Sys. 6 (1986), 1-8. MR 87j:58074

[24] M. Denker, C. Grillenberger and K. Sigmund, Ergodic theory on compact spaces, Lecture Notes in Math. 527, Springer, Berlin, 1976. MR 56:15879

[25] J. Fehrenbach and J. Los, Une minoration de l'entropie topologique des difféomorphismes du disque, preprint, 1993.

[26] G. L. Forti, L. Paganoni, and J. Smítal, Strange triangular maps of the square, Bull. Austral. Math. Soc. 51 (1995), 395-415. MR 96d:54036

[27] J. Franks and M. Misiurewicz, Cycles for disk homeomorphisms and thick trees, Contemporary Mathematics, 152 (1993), 69-139. MR 95e:58133

[28] A. Katok, Bernoulli diffeomorphisms on surfaces, Ann. of Math. 110 (1979), 529-547. MR 81a:28015

[29] Kloeden P. E., On Sharkovsky's cycle coexistence ordering, Bull. Austral. Math. Soc. 20 (1979), 171-177. MR 81d:58045

[30] S. F. Kolyada, On dynamics of triangular maps of the square, Ergod. Th. \& Dynam. Sys. 12 (1992), 749-768. MR 93m:58036

[31] S. F. Kolyada and L. Snoha, On $\omega$-limit sets of triangular maps, Real Analysis Exchange 18 (1992-93), 115-130. MR 94b:58057

[32] S. F. Kolyada and Ľ. Snoha, Topological entropy of nonautonomous dynamical systems, Random \& Computational Dynamics 4 (1996), 205-233. MR 98f:58126

[33] S. F. Kolyada and A. N. Sharkovsky, On topological dynamics of triangular maps of the plane, Proc. ECIT-89, World Scientific, Singapore (1991), 177-183. MR 93f:58196

[34] J. Llibre and M. Misiurewicz, Horseshoes, entropy and periods for graph maps, Topology 32 (1993), 649-664. MR 94k:58113

[35] J. Milnor, On the concept of attractor, Commun. Math. Phys. 99 (1985), 177-195. MR 87i:58109a

[36] M. Misiurewicz, Periodic points of maps of degree one of a circle, Ergod. Th. \& Dynam. Sys. 2 (1982), 221-227. MR 84j:58101 
[37] J. C. Oxtoby, Measure and category, Springer-Verlag, New York, 1971. MR 52:14213

[38] A. N. Sharkovsky, Co-existence of the cycles of a continuous mapping of the line into itself (in Russian), Ukrain. Math. Zh. 16 (1) (1964), 61-71.

[39] A. N. Sharkovsky, Nonwandering points and the centre of a continuous mapping of the line into itself (in Ukrainian), Dopovidi Ukrain. Acad. Sci. 7 (1964), 865-868.

[40] Ye. A. Sidorov, Smooth topologically transitive dynamical systems (in Russian), Mat. Zametki 4 (1968), 751-759.

[41] Ye. A. Sidorov, Topologically transitive cylindrical cascades (in Russian), Mat. Zametki 14 (1973), 441-452.

[42] B. Sivák, Strongly irreducible strings, Acta Univ. M. Belii, Ser. Math. 3 (1995), 81-87. MR 97g:20073

[43] R. F. Williams, The structure of Lorenz attractors, Lecture Notes in Math. 615, pp. 94-112, Springer, Berlin, 1977. MR 57:1566

Departament de Matemàtiques, Edifici Cc, Universitat Autònoma de Barcelona, 08193 - Bellaterra, Barcelona, Spain

E-mail address: alseda@mat.uab.es

Institute of Mathematics, Ukrainian Academy of Sciences, Tereshchenkivs'Ka 3, $252601 \mathrm{KIEV}-4$, UKRAINE

E-mail address: skolyada@math.carrier.kiev.ua

Departament de Matemàtiques, Edifici Cc, Universitat Autònoma de Barcelona, 08193 - Bellaterra, Barcelona, Spain

E-mail address: jllibre@mat.uab.es

Department of Mathematics, FPV, Matej Bel University, Tajovského 40, 97401 Banská Bystrica, Slovakia

E-mail address: snoha@bb.sanet.sk 